\title{
Brain Mechanisms for Detecting Perceptual, Semantic, and Emotional Deviance
}

\author{
B. A. Strange,* R. N. A. Henson,*'† K. J . Friston,* and R. J . Dolan*'‡ \\ *Wellcome Department of Cognitive Neurology, I nstitute of Neurology, 12 Queen Square, London WC1N 3BG, United Kingdom; \\ †I nstitute of Cognitive Neuroscience, 17 Queen Square, London WC1N 3BG, United Kingdom; and ¥Royal Free Hospital \\ School of Medicine, Roland Hill Street, London NW3 2PF, United Kingdom
}

Received February 21, 2000

The observation that we note the exceptional over the mundane has been the subject of extensive psychological and electrophysiological analysis in "oddball" paradigms. Whether detection of a sensory oddball reflects the operation of a generic mechanism or, alternatively, mechanisms sensitive to specific attributes of stimulus deviance is unknown. To address this question we used event-related functional MRI (fMRI) to measure neural responses during presentation of nouns, of which a proportion were perceptually, semantically, or emotionally deviant. Oddballs, regardless of deviant attributes and depth of processing, activated right inferior prefrontal and bilateral posterior fusiform cortices. Attribute-specific responses, independent of depth of processing, were evident in bilateral fusiform cortices for perceptual oddballs and left amygdala for emotional oddballs. By contrast, an interaction with depth of processing was evident in left prefrontal cortex for semantic oddballs. We conclude that detection of oddballs reflects the operation of a generic "deviance detection system," involving right prefrontal and fusiform cortices in addition to specific brain regions sensitive to the stimulus attributes that determine the qualitative characteristics of deviance. 2000 Academic Press

Key Words: oddball; functional MRI; perception; semantics; emotion; von Restorff memory effect; attention orienting; inferior parietal lobule; fusiform cortex; prefrontal cortex; amygdala.

Many neuropsychological studies of cognitive event-related potentials (ERPs) have focused on the P300 complex, evoked by the presentation of "oddball" stimuli (Rugg, 1995). However, the functional significance and neural origin of this oddball-evoked activity remain controversial. Hypotheses range from a single cognitive operation arising from activity in a single generator (Donchin and Coles, 1988) to the $\mathrm{P} 300$ reflecting summation of activity from multiple functionally independent generators, each sen- sitive to a different stimulus attribute (J ohnson, 1993). Using event-related fMRI we tested these hypotheses by presenting three types of oddballs, perceptual, semantic, and emotional, all of which evoke a P300 (Williamson et al., 1991; Fabiani and Donchin, 1995). Specifically, we tested for attributespecific neural responses as well as responses common to all three oddball types. Oddball-evoked neural responses also speak to mechanisms mediating enhanced memory for P300-evoking oddball stimuli, referred to as the von Restorff effect (von Restorff, 1933).

During fMRI scanning, 11 subjects viewed lists of 19 nouns, serially presented, where all nouns within a given list bel onged to the same category except for one, the semantic oddball. In these lists a further noun was presented in a novel font (perceptual oddball) and a further noun was emotionally aversive (emotional oddball). The three oddball types were randomly positioned within the lists with the constraints that the first five nouns were control nouns (i.e., nonoddballs), to set the context, and that each oddball was followed by at least one control noun. Lists were studied under one of two tasks: deep (requiring a living/nonliving judgement) and shallow processing (determining whether the first letter had an enclosed space) (Craik and Lockhart, 1972). We hypothesized that neural responses to the perceptual and emotional oddball would be task-independent, whereas neural responses to the semantic oddball would be enhanced by attending to meaning in the deep encoding condition. The P300 evoked by semantic oddballs is larger when subjects process stimuli according to semantic attributes than when attending to physical features (Fabiani and Donchin, 1995). Neural responses to semantic oddballs also address the origin of the N400 event-related potential, a component sensitive to semantic deviance that is also enhanced by semantic processing (Kutas and Van Petten, 1994). Figure 1 gives examples of the stimuli. 


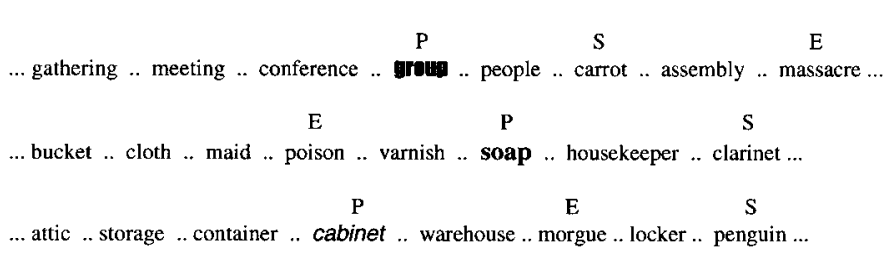

FIG. 1. Examples of presented nouns. Abbreviations: $P$, perceptual oddball; S, semantic oddball; E, emotional oddball.

\section{MATERIALS AND METHODS}

\section{Subjects}

Informed consent was obtained from 12 righthanded, native English speaking subjects ( 6 male, 6 female; age range 18-30 years; mean age 23.3). One subject was discarded due to technical failure. A further 10 subjects ( 3 male, 7 female; age range 22-32 years; mean age 26.3) completed the psychological task outside of MRI scanning.

\section{Psychological Task}

During scanning subjects viewed nouns presented visually in lower case at a rate of one every $3 \mathrm{~s}$. During each of the four sessions, subjects were presented with eight lists of 19 nouns with the words "New List" presented between lists. For each list, 16 nouns were of the same semantic category, emotionally neutral, and all presented in the same font. These are referred to as control nouns. The perceptual oddball was presented in a novel font but was emotionally neutral and of the same semantic category as the control words. The semantic oddball was of a different category but emotionally neutral and presented in the same font as the 16 control nouns. The emotional oddball was emotionally aversive but of the same category and perceptually identical to the control nouns. The semantically related nouns were constructed using the Edinburgh associative thesaurus (www.itd.clrc.ac.uk/Activity/Psych). Nouns were presented in Times font (48 point; $4-10^{\circ}$ horizontal visual angle) except for the perceptual oddballs, which appeared in 16 different fonts.

Subjects engaged in two distinct encoding tasks. During two of the sessions, subjects were required to indicate with a push-button whether or not the first letter in the noun had an enclosed space (the shallow encoding task) (Craik and Lockhart, 1972). During the other two sessions subjects indicated whether the noun described a living or nonliving entity (the deep encoding task). Encoding instructions were provided visually at the start of each session and half of the subjects followed the order: shallow, deep, deep, shallow, and the other half: deep, shallow, shallow, deep. For the 10 subjects who completed the psychological task outside of scanning, memory for presented nouns was assessed by verbal recall following the presentation of each list of 19 nouns. Due to constraints of $\mathrm{fMRI}$ scanning, it was not possible to assess recall after each list presentation for the subjects who were scanned.

In discussing our oddball-induced activations we refer to the P300 ERP. The P300 complex has been divided on the basis of scalp topography and task correlates into the frontocentral P3a, evoked by novel distractor stimuli and a component of the characteristic response to orienting stimuli, and a later parietal P3b, evoked by infrequent target stimuli (Rugg, 1995). Our experiment was not designed to dissociate these two components. F urthermore, the P300 evoked by an oddball stimulus is often preceded by a negative component (N1 and/or N2) and often followed by slow potential shifts (Alexander et al., 1997). These effects may contribute to the slow haemodynamic responses we observe.

\section{Data Acquisition}

A Siemens VISION system (Siemens, Erlangen), operating at $2 \mathrm{~T}$, was used to acquire both T1-weighted anatomical images and gradient-echo echoplanar T2*weighted MRI image volumes with blood oxygenation level dependent (BOLD) contrast. For each subject, data were acquired in four scanning sessions. A total of 540 volumes were acquired per subject plus 20 "dummy" volumes (5 at the start of each session), subsequently discarded, to allow for T1 equilibration effects. Volumes were acquired continuously every 3740 ms. Each volume comprised forty-two 3-mm axial slices, with an in-plane resolution of $3 \times 3 \mathrm{~mm}$, positioned to cover the whole brain. The imaging time series was realigned to correct for interscan movement and normalized into a defined space (Talairach and Tournoux, 1988) to allow group analyses. The data were then smoothed with a Gaussian kernel of $8 \mathrm{~mm}$ full-width half-maximum to account for residual intersubject differences (Friston et al., 1995).

\section{Data Analysis}

Data were analyzed using Statistical Parametric Mapping (SPM98) employing a random effects model implemented with a two level procedure. To test for the effects of each oddball type vs control we specified six effects of interest: the events corresponding to the presentation of the three oddball types and three randomly selected control nouns (one for each oddball type). These effects were model ed by convolving a delta function at each event onset with the haemodynamic response function, and its temporal derivative, to create regressors of interest. The events corresponding to the presentation of the "New List" marker were modeled as regressors of no interest, as were low frequency drifts in signal (cut-off $120 \mathrm{~s}$ ). The data were normalized for global effects by proportional scaling. The random effects analysis involved two stages. First, ses- 
sion-specific parameter estimates pertaining to the height of the haemodynamic response for each effect of interest were calculated for each voxel. An appropriate contrast of parameter estimates across sessions was calculated in a voxel-wise manner to produce, for each subject, one contrast image for that particular comparison. Secondly, six contrast images for each subject (one image for each oddball type and one for each control noun type, all six collapsed across deep and shallow encoding) were entered into a repeated measures ANOVA (with pooled error term) across the 11 subjects. Although this analysis collapsed across encoding task, the parameter estimates for reported activations are plotted for deep and shallow encoding tasks separately. The error bars in these plots depict the standard error of the mean of the parameter estimate differences, not the pooled error term. To test for oddball $\times$ encoding task interactions, a further six contrast images for each subject (one image for each oddball type minus respective control in the deep and in the shallow encoding conditions) were entered into a repeated measures ANOVA (with pooled error term) across the 11 subjects. This analysis also enabled us to search for an interaction with encoding task in regions that previously demonstrated oddball-evoked responses. The absence of a significant interaction $(\mathrm{P}<$ 0.05 uncor rected) in these regions suggested that oddball-evoked activation in these regions was independent of encoding task. Voxel-level correction accounted for spatial correlations between voxels according to the theory of Gaussian fields (Friston et al., 1994). We report all regions which survive correction at $\mathrm{P}<0.05$ plus those regions surviving an uncorrected threshold of $\mathrm{P}<0.001$ for which we had an a prior hypothesis for their activation (namely left and right prefrontal and inferior parietal cortices and amygdala). In all random effects ANOVAs it was assumed that the within-subject between-contrast variability was at the same level as the between-subject within-contrast variability for all pairs of contrasts (i.e., sphericity).

To examine responses commonly evoked by all oddballs, we carried out a conjunction analysis on the three oddballs versus their respective controls. The conjunction is defined as a significant main effect in the absence of any interactions among the simple effects (Price and Friston, 1997) and requires an independent baseline for each effect being tested, hence why three control words were randomly assigned as the control for each oddball type. In both reported conjunctions the interactions were removed at a threshold of $\mathrm{P}<0.001$. The conjunction therefore tests for the activating effects of oddballs relative to controls (or the modulatory effect of the right prefrontal cortex during presentation of oddballs versus controls) that are common to three different contexts. The analysis testing for the modulatory effects of the right prefrontal cortex (Friston et al., 1997) followed the same two-level procedure except that, for each subject, the effects of interest were the convolved oddball and control noun regressors multiplied by that subject's session-specific adjusted activity at, or nearest to, voxel $(48,21,21)$. The regressors themselves and the adjusted activity were modeled as effects of no interest.

\section{RESULTS}

\section{fMRI Data}

For each oddball type, we compared the neural response evoked by an oddball with that evoked by a randomly chosen control noun in the same list, yielding an independent control for each oddball. The chosen control nouns, like the oddballs, could not occur within the first five nouns of each list and could not immediately follow an oddball or another chosen control noun. To determine activation commonly evoked by all oddball types, we conducted a conjunction analysis of the three oddball types versus their respective controls. Activation, independent of encoding task, was evoked in right inferior frontal sulcus and bilateral posterior fusiform cortex (Fig. 2). The graph in Fig. 2 demonstrates the parameter estimates for the height of the haemodynamic response for the three oddballs minus their respective control nouns in right prefrontal cortex for both deep and shallow encoding. Right prefrontal activation evoked by oddballs, irrespective of the attribute conferring deviance, is consistent with a role for this region in monitoring discrepancies between expectation and experience (Fink et al., 1999).

It is thought that oddball detection involves recruitment of an attentional orienting system (Ritter et al., 1968). A monitoring role for right prefrontal cortex suggests its involvement in engaging attention during oddball presentation. Consequently, we created a statistical parametric map to identify areas where activity can be explained in terms of oddball-specific interactions with right prefrontal activity. The analysis uses a statistical model that includes an effect of each oddball type, an effect of each corresponding control noun and a term that represents the interaction between each of these effects and the adjusted activity in the right prefrontal cortex. A conjunction analysis of these interactions for all three oddball types revealed significant modulatory effects in inferior parietal lobule (IPL) bilaterally (Fig. 3). This prefrontal-parietal coupling was not significantly modulated by depth of processing.

In addition to a right prefrontal-fusiform generic deviance detection network, we demonstrate attributespecific responses that are evoked in neuroanatomical regions known to be sensitive to either the perceptual, semantic or emotional content of stimuli. The neural response to perceptual oddballs, relative to their controls, is shown in Fig. 4 a and demonstrates activation in posterior fusiform cortices bilaterally $(\mathrm{P}<0.05$ cor- 

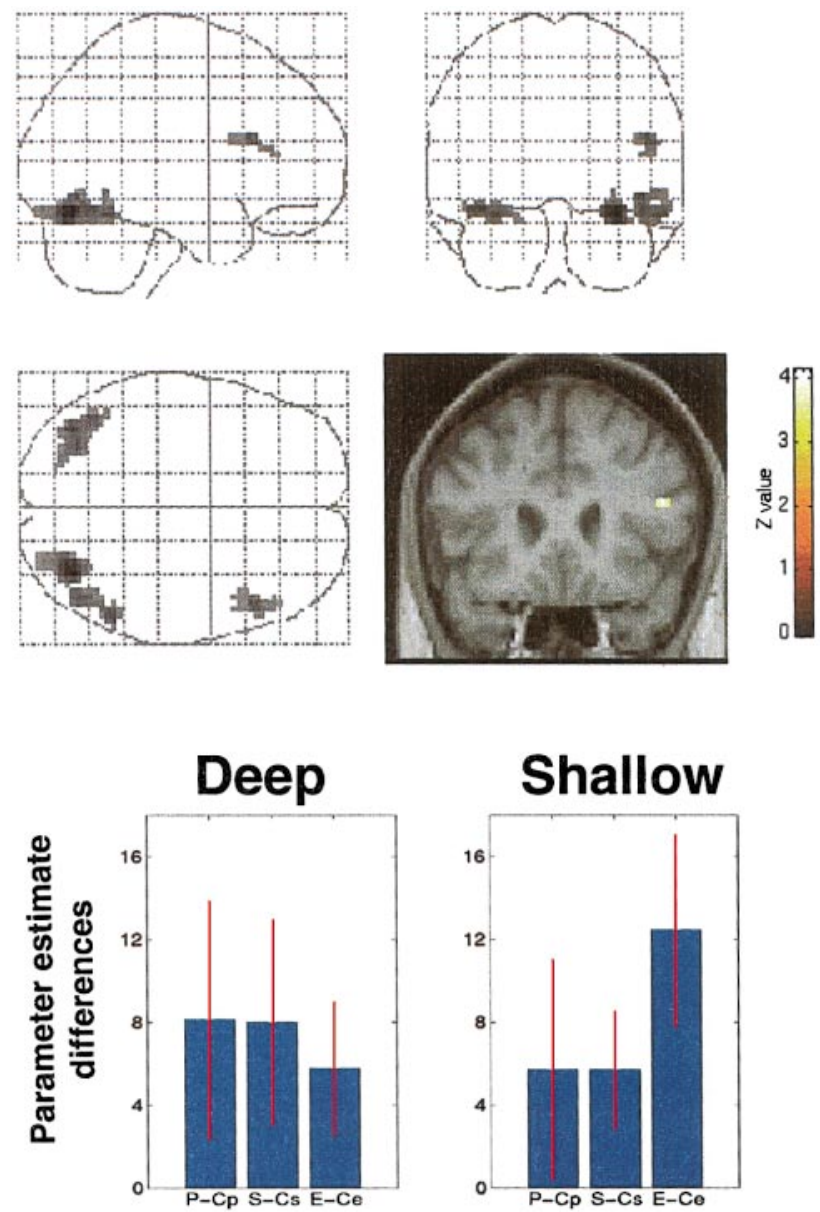

\section{Shallow}

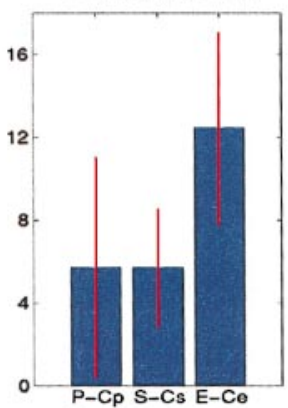

FIG. 2. Areas commonly activated by all oddball types. The SPM (threshold $\mathrm{P}<0.001$, extent threshold $\mathrm{P}<0.05$ ) of the conjunction is superimposed on a glass brain and demonstrates activation in the right prefrontal cortex (dorsal BA45; x, y, z coordinates 48, 21, 21; $Z=4.15)$ and bilateral posterior fusiform $(33,-75,-18 ; Z=5.51$, $P<0.05$ corrected and $-39,-72,-15 ; Z=4.42$, both in BA19) extending into the inferior temporal gyrus on the right. Also shown is the SPM superimposed on a coronal section of a normalised canonical T1 image at $y=21$, demonstrating activation in right inferior frontal sulcus. Note that a localized region of activation, not surviving the extent threshold, was observed in the right anterior prefrontal cortex in superior frontal gyrus (BA9; 12, 57, 36; $Z=4.88$, $\mathrm{P}<0.05$ corrected). The plot shows the parameter estimates for the height of the haemodynamic response in right prefrontal cortex for the three oddballs minus their respective control words during both deep and shallow encoding. The units are adimensional and correspond to responses per unit increase in the explanatory variables scaled arbitrarily. Here and in all subsequent figures, error bars represent \pm 1 standard error and abbreviations are: $\mathrm{P}$, perceptual oddball; S, semantic oddball; $E$, emotional oddball; $C p$, control noun for perceptual oddball; Cs, control noun for semantic oddball; $\mathrm{Ce}$, control noun for emotional oddball.

rected) extending into inferior temporal gyrus. The response to perceptual oddballs is not significant modulated by depth of encoding, despite the shallow task directing attention to the perceptual attributes of the nouns, suggesting that the fusiform response to perceptual novelty is automatic and independent of the aspect of the stimulus being processed. The plots also demonstrate that the posterior fusiform is sensitive to semantic and emotional oddballs, as indicated by the conjunction analysis of all oddball types versus control.

Figure 4b illustrates the neural responses to emotional oddballs relative to controls. Activation was observed in left amygdala ( $P<0.001$, Fig. $4 b(i))$ and in left inferior prefrontal cortex $(P<0.05$ corrected, Fig. $4 b(i i))$. I mportantly, as for perceptual oddballs, the responses in left amygdala and inferior frontal cortex appear automatic and obligatory in so far as they are not modulated by depth of processing.

Although the above responses were task-independent, we predicted that semantic oddballs would show enhanced responses only when subjects attended to the semantic characteristics of stimuli. This interaction of deep versus shallow encoding and semantic oddballs versus controls was evident in left ventral prefrontal cortex, on the inferior bank of the inferior frontal sulcus (Fig. 4c).

\section{Behavioral Data}

Figure 5 illustrates free recall for oddballs versus control nouns, which was assessed in a separate group
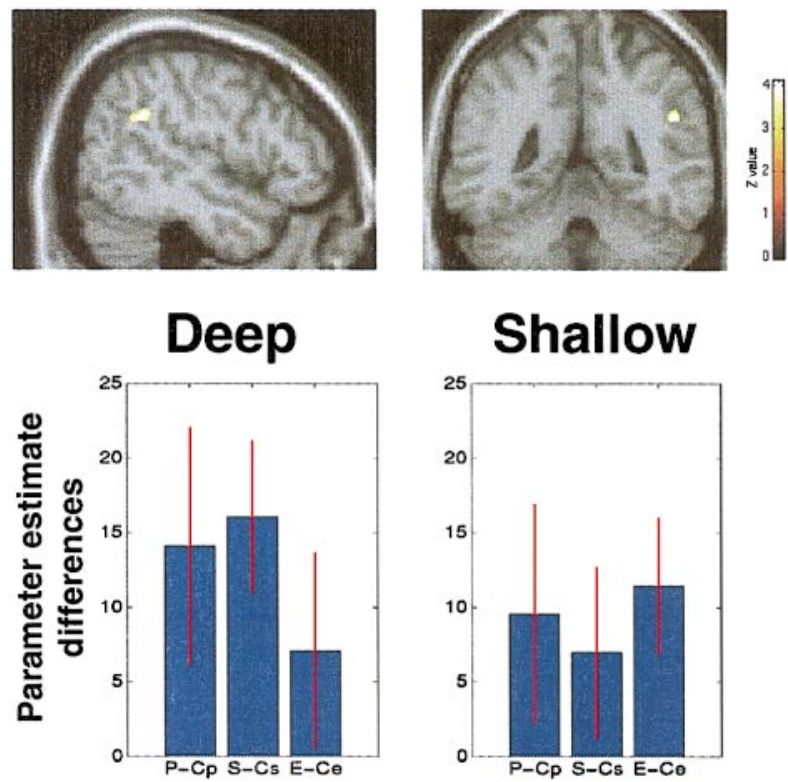

FIG. 3. The right prefrontal cortex exerts significant modulatory effects on activity in the right inferior parietal lobule during presentation of all oddball types relative to control nouns. The SPM (threshold $P<0.001)$ is superimposed on a saggital section at $x=51$ and coronal $(y=-45)$ of the T1 image and demonstrates activation of the right inferior parietal Iobule (BA 40; 51, $-45,30 ; Z=4.11$ ). Activation was also observed in left inferior parietal lobule (BA 40; -48 , $-57,36 ; Z=3.79$ ). The plot demonstrates the parameter estimates for the degree to which the height of the right inferior parietal haemodynamic response is modulated by the right prefrontal cortex during presentation of each oddball relative to respective control nouns during both deep and shallow encoding. 
a

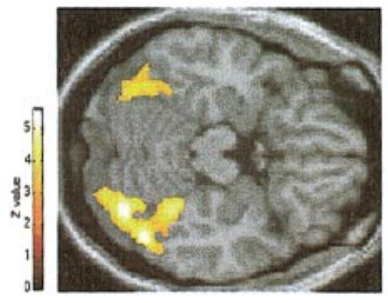

b

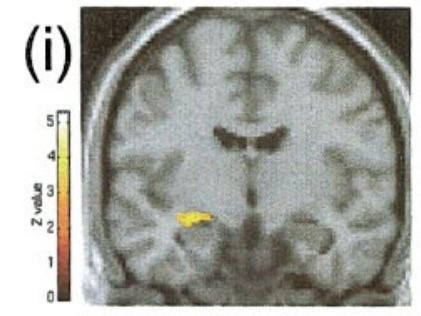

(ii)

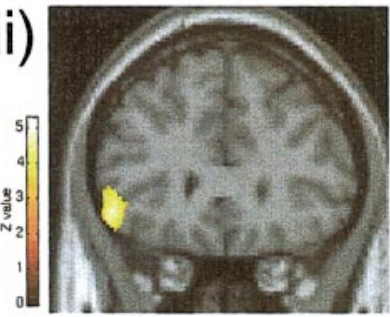

C

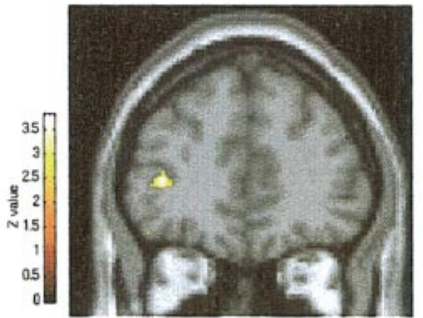

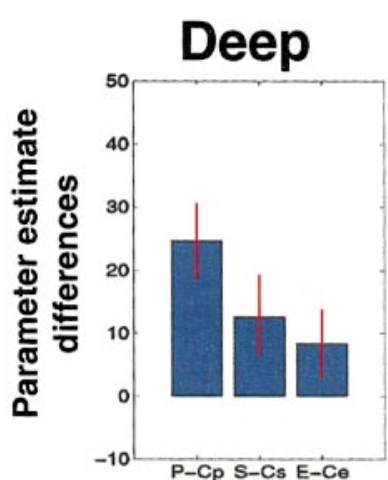
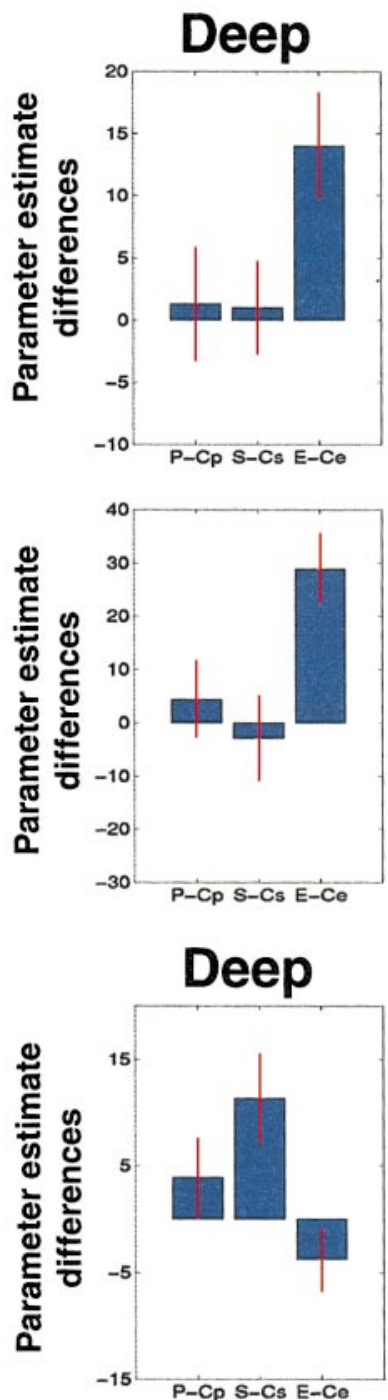

Shallow

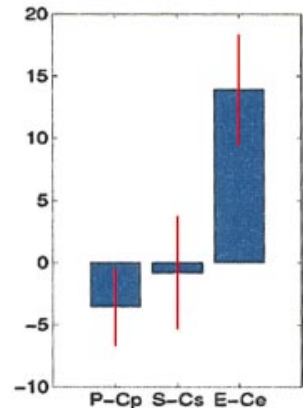

Shallow
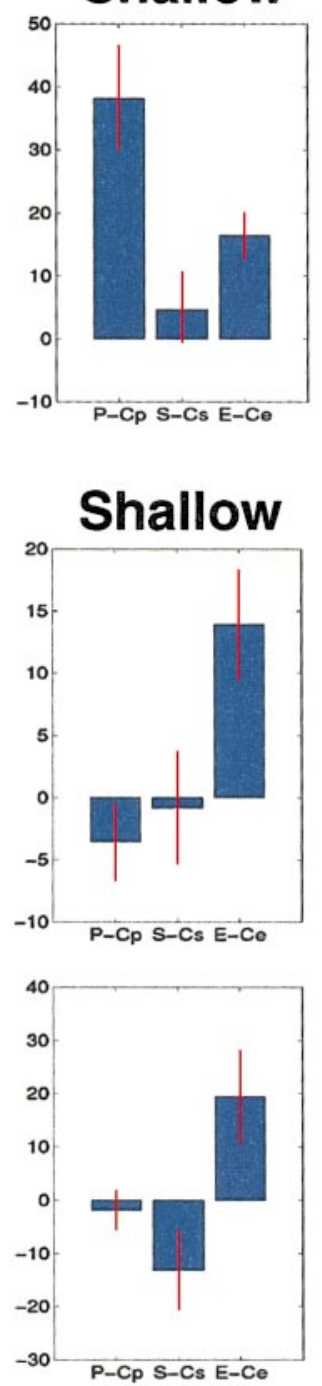

Shallow

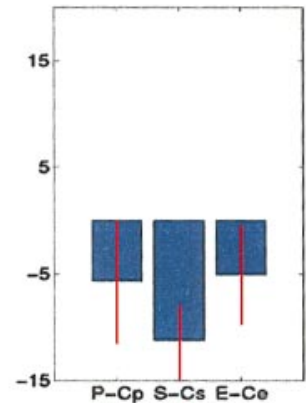

FIG. 4. Attribute-specific activations. (a) Perceptual oddballs, regardless of encoding task, activate the posterior fusiform cortices bilaterally $(33,-75,-18 ; Z=5.66$ and $-45,-63,-15 ; Z=4.95$ both in BA19 and both $P<0.05$ corrected for multiple comparisons) extending into inferior temporal cortex. The SPM, thresholded at $\mathrm{P}<0.001$ and superimposed on a transverse section of the canonical T1 image $(z=-18)$, demonstrates this activation. The parameter estimates for the height of the response in right posterior fusiform during both deep and shallow encoding are shown to the right of the section. (b) E motionally aversive oddballs activate the left amygdala and left inferior frontal cortex, regardless of encoding task. (i) The SPM (threshold P $<0.001$ ) is superimposed on a coronal section of the T1 image at $y=$ -9 and demonstrates activation of the left amygdala $(-27,-9,-12 ; Z=4.14)$. (ii) The same SPM is superimposed on a coronal section ( $y=$ 36) and shows activation of the left inferior prefrontal cortex (BA47; $-51,36,-12 ; Z=5.38, P<0.05$ corrected). (c) The response to semantic oddballs is modulated by depth of processing. Left, the SPM (threshold P $<0.01$ ) superimposed on a coronal section of the T1 image at $\mathrm{y}=$ 42 demonstrates the interaction of deep vs shallow encoding of the semantic oddballs relative to control in left ventral prefrontal cortex, on the inferior bank of the inferior frontal sulcus (BA46; $-36,42,9 ; Z=3.88$ ). 


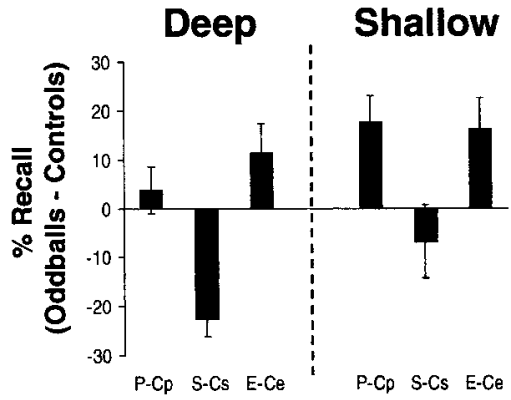

FIG. 5. Recall performance for the 10 subjects who completed the psychological task outside of scanning, recalling nouns freely after each 19-noun list presentation. The difference between recall of each oddball type and its respective control noun is plotted following both deep and shallow encoding. Subjects demonstrated enhanced recall for perceptual oddballs relative to controls (i.e., a von Restorff effect) following shallow encoding (one-tailed t test; $\mathrm{P}<0.01$ ), but not following deep encoding ( $P>0.2$ ). Recall of semantic oddballs was not significantly different from controls following shallow encoding $(P>0.15)$, but deep encoding resulted in significantly less semantic oddballs being recalled than controls $(P<0.005)$. A von Restorff effect was evident for emotional oddballs following both deep ( $P<$ $0.05)$ and shallow $(P<0.05)$ encoding. Error bars represent \pm 1 standard error.

of 10 subjects. Recall was tested immediately after each 19-noun list under both deep and shallow encoding conditions. We demonstrate that the von Restorff effect is influenced by both attribute deviancy and the encoding task. Emotional oddballs were remembered better than controls regardless of encoding task. Perceptual oddballs, however, only showed a von Restorff effect for shallow-encoded nouns. Semantic oddballs show no von Restorff effect following shallow encoding and are in fact recalled worse than controls following deep encoding. The mean percentage of recalled control nouns following deep encoding was $60.8 \%$ and following shallow encoding was $50 \%$.

\section{DISCUSSION}

Right prefrontal activation evoked by oddballs, irrespective of the attribute conferring deviance, is consistent with a role for this region in monitoring discrepancies between expectation and experience. The activation in right inferior frontal sulcus lies between the right ventral prefrontal region we have previously found responsive to sensory conflict between vision and proprioception and the right dorsal prefrontal region we found responsive when maintaining action in the face of conflict between intention and sensory outcome (Fink et al., 1999). Furthermore, a previous neuroimaging study of visual oddballs (McCarthy et al., 1997) reported bilateral activation of middle frontal gyrus, with more extensive activation on the right. A role for the right prefrontal-bilateral fusiform network in processing generic deviance is supported by a similar network being engaged by viewing abnormally coloured objects compared to viewing their black and white counterparts (Zeki and Marini, 1998).

Two previous functional imaging studies of visual (McCarthy et al., 1997) and auditory (Higashima et al., 1996) oddball detection have demonstrated oddballevoked activation of IPL. The former study suggested that bilateral IPL activation reflected the engagement of working memory whereas the latter suggested that right IPL mediates auditory discrimination. We suggest instead that the activation of IPL, which was most significant on the right, reflects increased attention to oddballs relative to control nouns. Note that the right hemisphere, particularly the IPL, is thought to be critical for human attention (Heilman et al., 1985; MesuIam, 1990; Driver and Mattingley, 1998). Our data suggest that a discrepancy detector located in the right prefrontal cortex mediates this attentional engagement.

It has been suggested that activity in the inferior frontal sulcus and IPL mediate a "preparation-to-process" function of the orienting response (Baudena et al ., 1995; Halgren et al., 1995; Halgren and Marinkovik, 1995), a component of which is manifest electrophysiologically as the P300 evoked by novel stimuli (Halgren and Marinkovik, 1995). The orienting response prepares an organism to process (via arousal and directed attention) and react to biologically prepotent stimuli. Although the IPL makes a major contribution to the P300 (Smith et al., 1990; Halgren et al., 1998), the P300 evoked by novel stimuli has a shorter latency in prefrontal than in parietal cortex (Baudena et al., 1995; Halgren et al., 1995) and prefrontal lesions attenuate this P300 component over both anterior and posterior cortex (Knight, 1984). This supports our observation of prefrontal modulation of IPL activity as well as the suggestion that prefrontal cortex plays a leading role in organizing the human orienting response (Halgren and Marinkovik, 1995). Furthermore, the observed prefrontal-IPL response to oddballs might explain why lesion studies report attenuation of the oddball-evoked P300 following either lesions to temporoparietal junction (TPJ, which includes the inferior parietal lobule) or prefrontal cortex (Knight et al., 1989; Yamaguchi and Knight, 1991). In further support of a prefrontal role in engaging attention, patients with frontal damage exhibit diminished visual attention to novel events (Daffner et al., 2000) leading to the suggestion that frontal damage prevents the generation of a signal indicating that a novel stimulus requires additional attention.

The posterior fusiform region activated by all oddball types has been shown to be equally sensitive to words and letter strings but insensitive to word meaning (Nobre et al., 1994). This suggests that posterior fusiform mediates a prelexical stage in word processing prior to semantic or emotional evaluation. This is consistent with our observation that perceptual oddballs 
evoke activation in bilateral posterior fusiform, indicating sensitivity to perceptual attributes of stimuli (Desimone and Ungerleider, 1989). Functional imaging data have shown that posterior fusiform mediates early preferential selection of attended visual stimuli (Heinze et al., 1994) with selective responses to infrequent targets (Coull et al., 1998). Furthermore, intracranial ERPs demonstrate that focusing attention on words evokes focal field potentials in posterior fusiform (Nobre et al., 1998). We propose, therefore, that activation in posterior fusiform reflects attentional modulation of this prelexical stage, yielding all threetypes of oddball nouns preferential access to higher stages of the word recognition process. In addition, therefore, to the specific fusiform role in processing perceptual features, we demonstrate modulation of a perceptual region by the semantic and emotional content of stimuli. Activation in posterior fusiform may, however, also reflect longer time spent attending to oddballs. It has been demonstrated that activity in bilateral posterior fusiform increases with increasing presentation duration of words (Price et al., 1996) and reaction times showed that subjects took longer to respond to oddballs than they did to controls (main effect of oddball significant at $P<0.05$, with no interactions reaching significance).

Human lesion data (Aggleton, 1992) and neuroimaging studies (Morris et al., 1996) indicate that the amygdala has a critical role in detecting and responding to stimuli that represent threat. A recent functional imaging study (I senberg et al., 1999) also demonstrates an amygdala response to visually presented threatening words. The left inferior frontal gyrus may also play a role in detecting threat as it has been shown to activate with increasing fearful intensity of faces (Morris et al., 1998). These activations may, however, be attributed to simple aversiveness rather than to emotional deviance from the current context. One way to dissociate these effects would be to measure neural responses commonly evoked by an aversive word presented in a neutral context and by a neutral word that is presented in an aversive context.

There exists evidence for greater episodic memory for emotionally aversive relative to neutral events (Cahill et al., 1996) and words (Bower, 1992), but whereas amygdala activation during encoding has been shown to correlate with subsequent retrieval of visual stimuli (Cahill et al., 1996), the same has yet to be demonstrated for emotional words. Our findings that, regardless of encoding task, the left amygdala is activated by emotionally aversive nouns and that these nouns are recalled better than their neutral controls, suggests that amygdala activity during encoding of emotional words would correlate with subsequent recall.

The responses evoked by perceptual and emotional oddballs were not significantly modulated by depth of processing suggesting automatic and obligatory re sponses. The left prefrontal response evoked by semantic oddballs was, however, significantly enhanced by attending to the semantic characteristics of nouns (deep encoding) relative to attending to their perceptual features (shallow encoding). This left prefrontal activation is commonly associated with memory encoding tasks, particularly those that emphasise processing of study material in terms of semantic attributes (Tulving et al., 1994; Dolan and Fletcher, 1997).

Perceptual oddballs were remembered better than controls following shallow but not deep encoding. This modulation of the von Restorff effect by depth of encoding could be due to the shallow task directing attention to the perceptual attributes of the nouns, yielding the perceptual deviance more memorable. Nonetheless, a previous study found a von Restorff effect for perceptual oddballs only following rote encoding, which does not direct attention towards perceptual attributes, and not following elaborative encoding (Fabiani et al., 1990). As suggested by Fabiani et al. (1990), the modulation we observe may also arise from differences in retrieval strategy following the two levels of processing. Although the perceptual oddball will be noted as deviant during both encoding tasks, only following shallow encoding might subjects use this perceptual attribute in their retrieval search. Deep encoding would encourage a category-cued retrieval strategy, however, making subjects less reliant on perceptual characteristics during encoding. That emotional oddballs are recalled better than control nouns following both encoding tasks suggests that the mnemonic advantage given to emotionally aversive stimuli is not modulated by encoding task or subsequent retrieval strategy. Semantic oddballs are recalled worse than controls following deep encoding, most likely reflecting an effect of the category-cued retrieval strategy adopted following deep encoding of the semantically related control nouns.

There is evidence that interactions between frontal and medial temporal perirhinal cortices mediate the von Restorff effect (Parker et al., 1998). Intracranial recordings (Halgren et al., 1980) and lesion studies (Knight, 1996) have implicated the hippocampus as a possible generator of the P300 but these results have been disputed (Polich and Squire, 1993) and previous functional imaging studies of oddball detection have failed to demonstrate hippocampal activation ( $\mathrm{Hi}-$ gashima et al., 1996; McCarthy et al., 1997). In our experiment, the lack of oddball-induced medial temporal lobe activation may have been due to the fact that all nouns were novel in the experimental context, yielding high baseline hippocampal activity (Dolan and Fletcher, 1997; Strange et al., 1999).

In conclusion, our data provide evidence in support of both hypotheses (Donchin and Coles, 1988; J ohnson, 1993) concerning the origin and function of oddballevoked neural activity. Oddball nouns engage a dis- 
crete attention orienting network as well as attributespecific, functionally independent brain structures. The activation of an attention network coupled with enhanced activity in neuroanatomical regions known to be sensitive to the perceptual, semantic, or emotional content of stimuli, may influence the von Restorff effect for oddballs. Importantly, however, we have shown that this memory effect is likely to depend on interactions between the attribute conferring deviance, the encoding task and the subsequent retrieval strategy.

\section{ACKNOWLEDG MENTS}

B.A.S. is supported by the Astor Foundation Scholarship. R.N.A.H., K.J .F., and R.J .D. are supported by the Wellcome Trust. We thank R. Frackowiak and L. Otten for internal review of this manuscript.

\section{REFERENCES}

Aggleton, J. P. 1992. The functional effects of amygdala lesions in humans: A comparison with findings from monkeys. In The Amygdala: Neurobiological Aspects of Emotion Memory and Mental Dysfunction (J . P. Aggleton, Ed.), pp. 485-503. Wiley-Liss, New York.

Alexander, J . E., Porjesz, B., Bauer, L. O., Kuperman, S., Morzorati, S., O'Connor, S. J., Rohrbaugh, J., Begleiter, H., and Polich, J. 1995. P300 hemispheric amplitude asymmetries from a visual oddball task. Psychophysiology 32: 467- 475.

Baudena, P., Halgren, E., Heit, G., and Clarke, J . M. 1995. Intracerebral potentials to rare target and distractor auditory and visual stimuli. III. Frontal cortex. Electroencephalagr. Clin. Neurophysiol. 94: 251-264.

Bower, G. H. 1992. How might emotions affect learning? In The Handbook of Emotion and Memory: Research and Theory (S-A. Christianson, Ed.), pp. 3-31. Lawrence Erlbaum, Hillsdale, NJ .

Cahill, L., Haier, R. J ., Fallon, J ., Alkire, M. T., Tang, C., Keator, D., Wu, J., and McGaugh, J. L. 1996. Amygdala activity at encoding correlated with long-term free recall of emotional information. Proc. Natl. Acad. Sci. USA 93: 8016-8021.

Coull, J. T., Frackowiak, R. S. J ., and Frith, C. D. 1998. Monitoring for target objects: Activation of right frontal and parietal cortices with increasing time on task. Neuropsychologia 36: 1325-1334.

Craik, F. I. M., and Lockhart, R. S. 1972. Levels of processing: A framework for memory research. J . Verbal. Learn. Verbal. Behav. 11: $671-684$.

Daffner, K. R., Mesulam, M. M., Scinto, L. F. M., Acar, D., Calvo, V., Faust, R., Chabrerie, A., Kennedy, B., and Holcomb, P. 2000. The central role of the prefrontal cortex in directing attention to novel events. Brain 123: 927-939.

Desimone R., and Ungerleider, L. G. 1989. Neural mechanisms of visual processing in monkeys. In Handbook of Neuropsychology ( $F$. Boller, and J. Grafman, Eds.), Vol. 2 pp. 267-296. Elsevier, New York.

Dolan, R. J ., and Fletcher, P. C. 1997. Dissociating prefrontal and hippocampal function in episodic memory encoding. Nature 388: 582-585.

Donchin, E., and Coles, M. G. H. 1988. Is the P300 component a manifestation of context updating? Behav. Brain. Sci. 11: 357-374.

Driver, J ., and Mattingley, J. B. 1998. Parietal neglect and visual awareness. Nature Neurosci. 1: 17-22.

Fabiani, M., and Donchin, E. 1995. Encoding processes and memory organisation: A model of the von Restorff effect. J . Exp. Psychol. Learn. Mem. Cogn. 21: 224-240.
Fabiani, M., Karis, D., and Donchin, E. 1990. Effects of strategy manipulation in a von Restorff paradigm. Electroencephalogr. Clin. Neurophysiol. 75: 22-35.

Fink, G. R., Marshall, J . C., Halligan, P. W., Frith, C. D., Driver, J ., Frackowiak, R. S. J., and Dolan, R. J. 1999. The neural consequences of conflict between intention and the senses. Brain 122: 497-512.

Friston, K. J ., Worsley, K. J ., Frackowiak, R. S. J ., Mazziotta, J . C., and Evans, A. C. 1994. Assessing the significance of focal activation using their spatial extent. Hum. Brain Mapp. 1: 214-220.

Friston, K. J ., Holmes, A., Worsley, K., Poline, J ., Frith, C., Heather, J ., and Frackowiak, R. S. J . 1995. Statistical parametric maps in functional imaging: A general linear approach. Hum. Brain Mapp. 2: $189-210$.

Friston, K. J ., Buechel, C., Fink, G. R., Morris, J., Rolls, E., and Dolan, R. J. 1997. Psychophysiological and modulatory interactions in neuroimaging. Neuroimage 6: 218-229.

Halgren, E., and Marinkovik, K. 1995. Neurophysiological networks integrating human emotions. In The Cognitive Neurosciences (M. Gazzaniga, Ed), pp. 1137-1151. MIT Press, Cambridge, MA.

Halgren, E., Squires, N. K., Wilson, C. L., Rohrbaugh, J. W., Babb, T. L., and Crandall, P. H. 1980. Endogenous potentials generated in the human hippocampal formation and amygdala by infrequent events. Science 210: 803- 805.

Halgren, E., Baudena, P., Clarke, J . M., Heit, G., Liegeois, C., Chauvel, P., and Musolino, A. 1995. Intracerebral potentials to rare target and distractor auditory and visual stimuli. I. Superior temporal plane and parietal lobe. Electroencephalogr. Clin. Neurophysiol. 94: 191-220.

Halgren, E., Marinkovik, K., and Chauvel, P. 1998. Generators of the late cognitive potentials in auditory and visual oddball tasks. Electroencephalogr. Clin. Neurophysiol. 106: 156-164.

Heilman, K. M., Watson, R. T., and Valenstein, E. 1985. Neglect and related disorders. In Clinical Neuropsychology (K. M. Heilman, and E. Valenstein, Eds.), pp. 243-293. Oxford UP, New York.

Heinze, H. J ., Mangun, G. R., Burchert, W., Hinrichs, H., Scholz, M., Münte, T. F., Gös, A., Scherg, M., J ohannes, S., Hundeshagen, H., Gazzaniga, M. S., and Hillyard, S. A. 1994. Combined spatial and temporal imaging of brain activity during visual selective attention in humans. Nature 372: 543-546.

Higashima, M., Kawasaki, Y., Urata, K., Maeda, Y., Sakai, N., Mizukoshi, C., Nagasawa, T., Kamiya, T., Yamaguchi, N., Koshino, Y., Matsuda, H., Tsuji, S., Sumiya, H., and Hisasda, K. 1996. Simultaneous observation of regional cerebral blood flow and event-related potential during performance of an auditory task. Cogn. Brain Res. 4: 289-296.

Isenberg, N., Silbersweig, D., Engelien, A., Emmerich, S., Malavade, K., Beattie, B., Leon, A. C., and Stern, E. 1999. Linguistic threat activates the human amygdala. Proc. Natl. Acad. Sci. USA 96: 10456-10459.

J ohnson, R., J r. 1993 On the neural generators of the P300 component of the event-related potential. Psychophysiology 30: 90-97.

Knight, R. T. 1984. Decreased response to novel stimuli after prefrontal lesions in man. Electroenceph. Clin. Neurophysiol. 59: 920.

Knight, R. T. 1996. Contribution of human hippocampal region to novelty detection. Nature 383: 256-259.

Knight, R. T., Scabini, D., Woods, D. L., and Clayworth, C. C. 1989. Contributions of temporo-parietal junction to the human auditory P3. Brain Res. 502: 109-116.

Kutas, M. and Van Petten, C. K. 1994. Psycholinguistics electrified: Event-related brain potential investigation. In Handbook of Psycholinguistics (M. A. Gernsbacher, Ed.), pp. 83-144. Academic Press, San Diego. 
McCarthy, G., Luby, M., Gore, J., and Goldman-Rakic, P. 1997. Infrequent events transiently activate human prefrontal and parietal cortex as measured by functional MRI. J. Neurophysiol. 77: $1630-1634$.

Mesulam, M. M. 1990. Large-scale neurocognitive networks and distributed processing for attention language and memory. Ann. Neurol. 28: 597- 613.

Morris, J . S., Frith, C. D., Perrett, D. I., Rowland, D., Young, A. W., Calder, A. J ., and Dolan, R. J . 1996. A differential neural response in the human amygdala to fearful and happy facial expressions. Nature 383: 812- 815.

Morris, J . S., Friston, K. J ., Buechel, C., Frith, C. D., Young, A. W., Calder, A.J ., and Dolan, R. J . 1998. A neuromodulatory rolefor the human amygdala in processing emotional facial expressions. Brain 121: 47-57.

Nobre, A. C., Allison, T., and McCarthy, G. (1994) Word recognition in the human inferior temporal lobe. Nature 372: 260-263.

Nobre, A. C., Allison, T., and McCarthy, G. 1998. Modulation of human extrastriate visual processing by selective attention to colours and words. Brain 121: 1357-1368.

Parker, A., Wilding, E., and Akerman, C. 1998. The von Restorff effect in visual object recognition memory in humans and monkeys: The role of frontal/perirhinal interactions. J . Cog. Neurosci. 10: 691-703.

Polich, J ., and Squire, L. R. 1993. P300 from amnesic patients with bilateral hippocampal lesions. Electroencephalogr. Clin. Neurophysiol. 86: 408-417.

Price, C. J ., and Friston, K. J . 1997. Cognitive conjunction: A new approach to brain activation experiments. Neuroimage 5: 261-270.

Price, C. J ., Moore, C. J ., and Frackowiak, R. S. J . 1996. The effect of varying stimulus rate and duration on brain activity during reading. Neuroimage 3: 40-52.
Ritter, W., Vaughan, H. G., J r., and Costa, L. D. 1968. Orienting and habituation to auditory stimuli: A study of short-term changes in average evoked responses. El ectroencephalogr. Clin. Neurophysiol. 25: 550-556.

Rugg, M. D. 1995. Cognitive event-related potentials: intracranial and lesion studies. In Handbook of Neuropsychology (F. Boller, and J. Grafman, Eds.), Vol. 10, pp. 165-185. Elsevier, New York.

Smith, M. E., Halgren, E., Sokolik, M., Baudena, P., Musolino, A., Liegeois-Chauvel, C., and Chauvel, P. 1990. The intracranial topography of the $\mathrm{P} 3$ event-related potential elicited during auditory oddball. Electroencephalogr. Clin. Neurophysiol. 76: 235-248.

Strange, B. A., Fletcher, P. C., Henson, R. N. A., Friston, K. J ., and Dolan, R. J . 1999. Segregating the functions of human hippocampus. Proc. Natl. Acad. Sci. USA 96: 4034- 4039.

Talairach, J ., and Tournoux, P. 1988. Co-planar Stereotaxic Atlas of the Human Brain. Thieme, Stuttgart.

Tulving, E., Kapur, S., Craik, F. I. M., Moscovitch, M., and Houle, S. 1994. Hemispheric encoding/retrieval asymmetry in episodic memory: Positron emission tomography findings. Proc. Natl. Acad. Sci. USA 91: 2016-2020.

von Restorff, H. 1933. Uber die wirkung von bereichsbildungen im spurenfeld [On the effect of spheres formation in the trace field]. Psychol. Forsch. 18: 299-342.

Williamson, S., Harpur, T. J., and Hare, R. D. 1991. Abnormal processing of affective words by psychopaths. Psychophysiology 28: $260-273$.

Yamaguchi, S., and Knight, R. T. 1991. Anterior and posterior association cortex contributions to the somatosensory P300. J . Neurosci. 11: 2039-2054.

Zeki, S., and Marini, L. 1998. Three cortical stages of colour processing in the human brain. Brain 121: 1669-1685. 